\title{
Orlans HO, Hornby SJ, Bowler IC (2011) In vitro antibiotic susceptibility patterns of bacterial keratitis isolates in Oxford, UK: a 10-year review. Eye Jan 21 [Epub ahead of print] PMID 21252952
}

\author{
Stephen Kaye $\cdot$ Rose Gilbert $\cdot$ Henri Sueke $•$ \\ Timothy Neal
}

Received: 8 March 2011 / Accepted: 9 March 2011 / Published online: 29 March 2011

(C) Springer-Verlag 2011

Bacterial keratitis remains a major cause of corneal opacity and loss of vision worldwide. Topical antimicrobial therapy is critical to its management. A number of antimicrobials are available to treat bacterial keratitis, with new antimicrobials occasionally introduced to provide an improved spectrum of activity against emerging or resistant pathogens. For example, cephalosporins were introduced in the 1960s as resistance of Staphylococcus aureus to penicillin became common, while gentamicin was introduced in the 1970s mainly due to an upsurge in Pseudomonas aeruginosa infections, associated with increasing contact lens wear use [1]. The first- and second-generation fluoroquinolones (ciprofloxacin and ofloxacin) have excellent potency against Gram-negative bacteria and reasonably good activity against the Gram-positive bacteria, with little corneal toxicity. When the third- and fourth-generation fluoroquinolones (levofloxacin, moxifloxacin and gatifloxacin) became available in the early 2000s, they offered a broader Gram-positive cover [1-3]. Despite the success of the first- and second-generation fluoroquinolones, there has been a trend in increased resistance from both S. aureus [4] and $P$. aeruginosa [5]. The fourth-generation fluoroquino-

S. Kaye $(\bowtie) \cdot$ R. Gilbert $\cdot$ H. Sueke

Department of Ophthalmology,

Royal Liverpool University Hospital,

Prescott Street,

Liverpool L7 8XP, UK

e-mail: s.b.kaye@liv.ac.uk

T. Neal

Department of Medical Microbiology,

Royal Liverpool University Hospital,

Prescott Street,

Liverpool L7 8XP, UK lones, moxifloxacin and gatifloxacin, have not unfortunately been a treatment panacea because of the emergence of resistance [6].

Deciding which antimicrobials to use for the treatment of bacterial keratitis can be challenging. It is important to be aware of the contemporaneous spectrum of likely causative bacteria, as this can vary according to the latitude and the degree of urbanisation of the population studied. Information regarding causative bacteria can only be attained by the collection of samples from the corneal ulcer and their subsequent analysis. Over time, a collection of such isolates provides knowledge not only on the likely bacteria, but also on their changing profiles against antimicrobial agents. This is also important, as treating physicians are often faced with an increasing choice of newer antimicrobial agents. The normal management strategy, if microbial infection is suspected, is to collect samples of corneal tissue for culture and then to initiate antimicrobial treatment empirically. The initial choice of treatment is either a single antimicrobial agent or a combination of antimicrobials providing a broad range of activity against both Gram-positive and Gramnegative bacteria. Once an isolate has been obtained, however, it is then possible to modify the treatment according to the in vitro susceptibility pattern of the isolate.

Orlans et al. [7] report on the spectrum of bacteria isolated from cases of keratitis in Oxford and the in vitro susceptibility data over a 10-year period. This type of study provides valuable information for ophthalmologists treating suspected bacterial keratitis in the clinical setting. In this study, they found that the most commonly isolated bacteria were Staphylococci (40.1\%), followed by Pseudomonas species $(28.5 \%)$, other Gram-negative species (17.2\%), Streptococci (7.1\%), and Corynebacteriacea (6.0\%), which 
is similar to previous studies [8]. Between the first and second time periods there was a significant increase in the number of coagulase-negative staphylococci (CNS). They also report that of the 189 isolates tested for sensitivity to their empirical antibiotic regimens, $93.2 \%$ were susceptible to ciprofloxacin, whereas $99.5 \%$ were susceptible to either gentamicin or cefuroxime.

It is important to be able to translate this type of information into clinical practice. One of the fundamental problems faced by ophthalmologists is deciding whether to change treatment, when faced with laboratory reports regarding susceptibility or resistance of the isolated bacteria, against the prescribed antimicrobial. It is, therefore, necessary to consider what is meant by resistance and susceptibility. These terms are used to indicate whether the infection caused by the isolated bacteria is likely to respond to a particular antimicrobial. This is inferred from the concentration of the antimicrobial drug that inhibits growth of the organism in the laboratory, that is, the minimum inhibitory concentration (MIC). If this concentration (MIC) is significantly less than the breakpoint concentration (a value determined by a number of factors including the concentration of the agent in the bloodstream), then it is usually reported as being susceptible to that antimicrobial.

MIC susceptibility breakpoints are based on the anticipated response of the bacteria against concentrations of the antimicrobial that can be achieved in serum. Topical application of an antimicrobial to the cornea may, however, achieve a very different tissue concentration and bioavailability than in the serum. The corneal penetration and effectiveness of a topical antimicrobial agent is dependent on the physicochemical properties of the antimicrobial and structure of the cornea. Furthermore, $\mathrm{pH}$ and protein binding of the local environment and interaction with other chemicals differ from systemic conditions, particularly in the inflamed eye. Topical administration of an antimicrobial to the eye involves mixing with the tear film, and so, loss of drug from the precorneal area is a net effect of corneal and non-corneal absorption, tear secretion, and drainage. Breakpoint concentrations have not been determined for topical antimicrobials, although there is evidence [9-11] demonstrating the relationship between MIC of topically applied antimicrobials and clinical outcome in bacterial keratitis. Previous work from our group has a demonstrated a direct relationship between bacterial susceptibility to a given antimicrobial in terms of MIC and reduction in healing time [11]. We found a significant association between the MIC of a fluoroquinolone and clinical outcome for Pseudomonas spp., S. aureus, and Enterobacteriaceae, but not for Streptococcus spp. or CNS. The absence of an apparent association between Streptococcus spp. and fluoroquinolone monotherapy may reflect the relatively poor activity of the fluoroquinolones against streptococci.
In contrast, evaluating the clinical significance of CNS isolates from samples is invariably difficult and the absence of an association for CNS may reflect the importance of host factors or simply that in a proportion of cases the CNS is not a pathogen. This is important, especially as Orlans et al. noted that between the first and second time periods there was an increase in the number of CNS strains isolated [7].

Interestingly, we have found that MICs were only associated with clinical outcome in $14-15 \%$ of cases of bacterial keratitis [11]. Therefore, although initiating prompt and appropriate topical antimicrobial therapy is essential to the management bacterial keratitis, it is clear that other factors, for example bacterial virulence factors, affect the clinical outcome. Also host factors, such as tear film composition, and mucin (glycoprotein) expression on the ocular surface, are likely to play a major role in determining resolution in microbial keratitis.

Orlans et al. describe a commonly used combination empirical therapy (cefuroxime and gentamicin) for the treatment of bacterial keratitis [7]. Combination therapy may offer a broader spectrum of activity [11] and reduce resistance selection pressures when compared to monotherapy; however, combinations of antimicrobials do not always result in synergistic or additive activity, and further studies on combination therapy for bacterial keratitis are required. The introduction of antimicrobials that are used increasingly to treat systemic infection into ophthalmic practice is an opportunity to improve treatment. It has been shown, for example, that an agent such as meropenem has excellent in vitro activity against commonly recognised pathogens [8]. In addition, the combination of meropenem and ciprofloxacin appear to be a good combination option (in vitro) against $S$. aureus and $P$. aeruginosa, with synergy in $20-25 \%$ and additivity in $55-60 \%$ of these isolates. Also against $S$. aureus, the combinations of teicoplanin with meropenem, ciprofloxacin, or moxifloxacin demonstrated either an additive or synergistic effect in more than $50 \%$ [12].

Susceptibility testing and monitoring of epidemiology is an important measure to evaluate the potential effectiveness of topically applied antimicrobials in the treatment of bacterial keratitis. A proof of principle, that is, the establishment that the outcome of bacterial keratitis is in part dependent on the MIC of the causative bacteria and prescribed antimicrobial, enables the determination of specific ophthalmic breakpoints without relying on systemic breakpoints, which has been the current practice. Such ophthalmic breakpoints can then be used to determine whether a given isolate is resistant to a given topical antimicrobial. In addition, we anticipate that identification of host factors influencing the clinical response in bacterial keratitis will be pivotal to pharmacological management in the future. Treatment strategies incorporating topical anti- 
microbials in combination with drugs to modify the ocular surface microenvironment may be a promising approach. These advances can facilitate ophthalmologists in their decisions regarding pharmacological treatment in bacterial keratitis to optimise clinical outcomes.

\section{References}

1. Baum J, Barza M (2000) The evolution of antibiotic therapy for bacterial conjunctivitis and keratitis: 1970-2000. Cornea 19:659672

2. Kowalski RP, Dhaliwal DK, Karenchak LM, Romanowski EG, Mah FS, Ritterband DC, Gordon YJ (2003) Gatifloxacin and moxifloxacin: an in vitro susceptibility comparison to levofloxacin, ciprofloxacin, and ofloxacin using bacterial keratitis isolates. Am J Ophthalmol 136:500-505

3. Mather R, Karenchak LM, Romanowski EG, Kowalski RP (2002) Fourth generation fluoroquinolones: new weapons in the arsenal of ophthalmic antibiotics. Am J Ophthalmol 133:463-466

4. Goldstein MH, Kowalski RP, Gordon YJ (1999) Emerging fluoroquinolone resistance in bacterial keratitis: a 5-year review. Ophthalmology 106:1313-1318

5. Garg P, Sharma S, Rao GN (1999) Ciprofloxacin-resistant Pseudomonas keratitis. Ophthalmology 106:1319-1323
6. Jhanji V, Sharma N, Satpathy G, Titiyal J (2007) Fourthgeneration fluoroquinolone-resistant bacterial keratitis. J Cataract Refract Surg 33:1488-1489

7. Orlans HO, Hornby SJ, Bowler IC (2011) In vitro antibiotic susceptibility patterns of bacterial keratitis isolates in Oxford, UK: a 10-year review. Eye Jan 21 [Epub ahead of print] PMID 21252952

8. Sueke H, Kaye S, Neal T, Murphy C, Hall A, Whittaker D, Tuft S, Parry C (2010) Minimum inhibitory concentrations of standard and novel antimicrobials for isolates from bacterial keratitis. Invest Ophthalmol Vis Sci 51:2519-2524

9. Wilhelmus KR, Abshire RL, Schlech BA (2003) Influence of fluoroquinolone susceptibility on the therapeutic response of fluoroquinolone-treated bacterial keratitis. Arch Ophthalmol 121:1229-1233

10. Chen A, Prajna L, Srinivasan M, Mahalakshmi R, Whitcher JP, McLeod S, Lietman TM, Acharya NR (2008) Does in vitro susceptibility predict clinical outcome in bacterial keratitis? Am J Ophthalmol 145:409-412

11. Kaye S, Tuft S, Neal T, Tole D, Leeming J, Figueiredo F, Armstrong M, McDonnell P, Tullo A, Parry C (2010) Bacterial susceptibility to topical antimicrobials and clinical outcome in bacterial keratitis. Invest Ophthalmol Vis Sci 51:362-368

12. Sueke H, Kaye SB, Neal T, Hall A, Tuft S, Parry CM (2010) An in vitro investigation of synergy or antagonism between antimicrobial combinations against isolates from bacterial keratitis. Invest Ophthalmol Vis Sci 51(8):4151-4155 